\title{
Utilization of the phase flicker of a LCoS Spatial Light Modulator for improved diffractive efficiency
}

\author{
Karol Kakarenko, * Marcin Zaremba, Izabela Ducin, Michał Makowski, Agnieszka Siemion, Andrzej Siemion, \\ Jarosław Suszek, Maciej Sypek, Dariusz Wojnowski, Zbigniew Jaroszewicz, Andrzej Kołodziejczyk
}

Faculty of Physics, Warsaw University of Technology, Koszykowa 75, 00-662 Warsaw

Received June 17, 2010; accepted September 12, 2010; published September 30, 2010

\begin{abstract}
The paper presents the observation, measurement and utilization of flickering of phase modulation in time on a high-end Liquid Crystal on Silicon (LCOS) Spatial Light Modulator. The flicker due to binary driving electronics is a negative effect, but can be overcome by appropriate adjustment of phase modulation depth, which results in time-synchronization of peak efficiencies for selected wavelengths. In this work optimal parameters for three wavelengths of primary RGB colors are investigated. The effect is optimal performance of the SLM for full-color dynamic holography.
\end{abstract}

We present preliminary experimental results of the measurement of phase flicker effects in a Holoeye PLUTO VIS spatial light modulator. This device provides pure-phase modulation for the entire visible wavelength range in a full $0-2 \pi$ phase range. Due to its small pixel pitch and a high fill factor of $87 \%$, the SLM is very suitable for dynamic holographic projection of color images [1-3]. Nevertheless, the price one pays for the 8bit phase shift addressing is the flicker effect caused by the driving electronics. The driver sends a sequence of binary signals causing the distribution of an electrical field throughout the pixels of the modulator. Due to the binary (on-off) scheme, the liquid molecules are always in movement, i.e. rotation of the LC director along the optimal orientation. This movement contributes to a fastchanging phase shift error of each pixel at a rate of approximately $120 \mathrm{~Hz}$. This effect is known [4, 5], but to our knowledge no investigation has been carried out taking into account the variable wavelength of a light incident on the SLM. In this contribution we examine the effect for the following wavelengths: $632.8 \mathrm{~nm}, 532 \mathrm{~nm}$ and $48 \mathrm{~nm}$ (from He-Ne, DPSS and Argon lasers, respectively).

The rapidly changing error in phase modulation contributes to changing diffractive efficiency, which was measured in our optical setup as shown in Fig. 1. A set of three binary diffractive gratings was displayed on the SLM - each grating dedicated to a different wavelength. The incident beams were converged on a common screen after an almost normal reflection from the SLM. In Fig. 1 only the red beam is shown for clarity. In the real setup the red, green and blue lasers were simultaneously illuminating appropriate parts of the SLM.

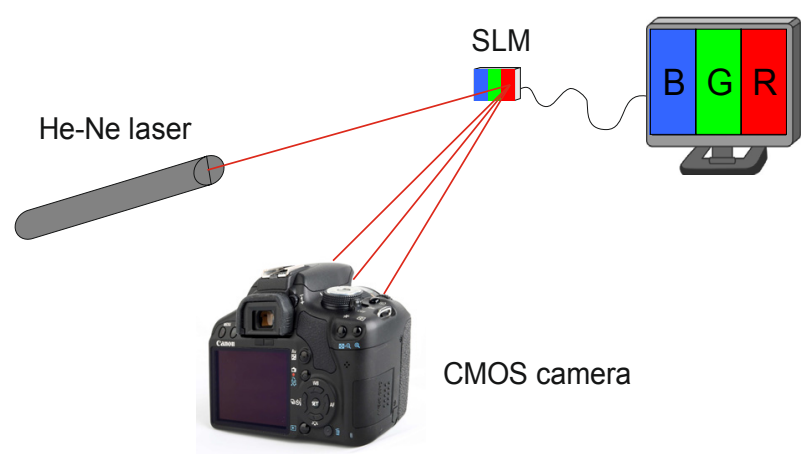

Fig. 1. Optical system for measuring the diffractive efficiency of diffractive gratings displayed on the SLM using a digital camera.

In this way we were able to change the grating depth individually for every beam. In order to capture fast oscillations a CMOS camera with very short exposure times was used, namely a Canon EOS 500D with an integration time of $1 / 1000 \mathrm{~s}$. By using the LiveView function of the camera we were able to capture subsequent exposures in a pace of 30 frames per second. The diffractive efficiency was evaluated by the measurement of brightness of recorded spots of light, i.e. the $0^{\text {th }},+1^{\text {st }}$ and $-1^{\text {st }}$ diffractive orders. The brightness value of a light spot in a particular order was divided by the sum of brightness values in three orders giving the approximate diffractive efficiency, normalized to $100 \%$. In this experiment a significant error was introduced by the non-linear characteristics of the camera for different colors, along with the possibility of light saturation of several pixels during the exposure. Nevertheless this amount of error was acceptable, because in this part of the experiment we wanted to establish the timecharacteristics of flickering for three wavelengths, not the absolute values of diffractive efficiencies. The values calculated for several periods of SLM addressing are shown in Fig. 2 and Fig. 3, in a time scale of consecutive frames collected by the digital camera. The graphs present an averaged relative diffractive efficiency in a particular 
diffractive order calculated as the sum of intensity in first orders divided by the sum of intensity in the lowest three orders (namely $+1^{\text {st }},-1^{\text {st }}$ and $0^{\text {th }}$ ). We decided to use the name "relative efficiency" because higher diffractive orders were not taken into account, thus introducing a systematic error. Therefore the achieved values are higher than real diffractive efficiency values in this configuration. Additionally, the efficiency for a binary grating in Fig. 3 is calculated as a sum of intensities in $+1^{\text {st }}$ and $-1^{\text {st }}$ orders divided by the sum of intensities in $+1^{\text {st }},-1^{\text {st }}$ and $0^{\text {th }}$ orders of diffraction. Please note that the goal of this measurement was not to indicate the absolute values of diffractive efficiency, but to measure the desynchronization in the phase fluctuations for different wavelengths.

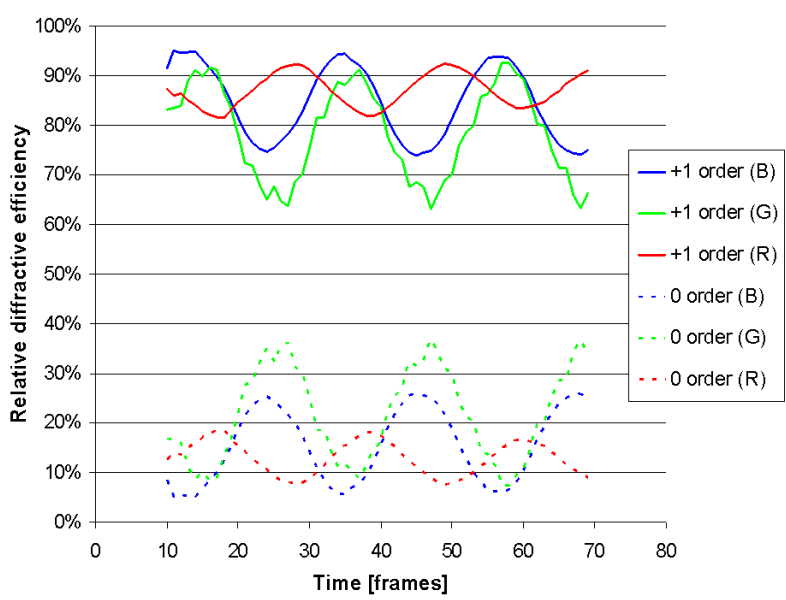

Fig. 2. Relative diffractive efficiency in particular diffractive orders for different wavelengths. Saw blade gratings were displayed on the SLM

with modulation depths established individually for the three wavelengths. Strong desynchronization of fluctuations is visible.

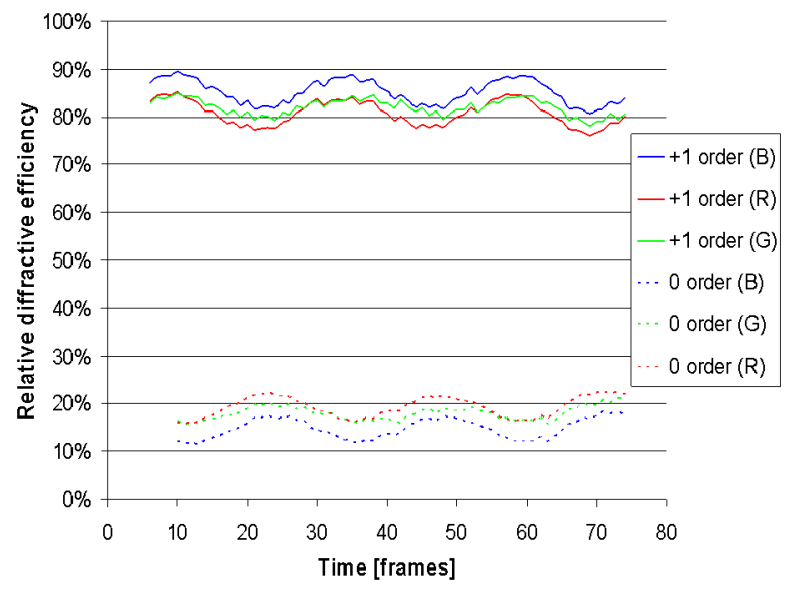

Fig.3. Diffractive efficiency in particular diffractive orders for different wavelengths. Binary gratings displayed on the SLM with modulation depths established commonly for the three wavelengths in order to achieve synchronization of fluctuations.
The fitted lines are 10-period average showing the mutual shift of the position of time slots of maximal efficiency for three wavelengths. When the three gratings addressed on the SLM have the optimal phase depth for each wavelength, providing the maximal efficiency, the mutual shift is large. We established experimentally the optimal combination of phase depth values for RGB beams, namely: 54 for $\mathrm{R}, 36$ for $\mathrm{G}$ and 37 for $\mathrm{B}$ (out of 256 possible phase levels). This combination provided the most correlated oscillations of diffractive efficiency simultaneously for three wavelengths. The results are gathered in Fig. 3. One can easily see the synchronization of the time characteristics for three beams. Therefore by using this simple procedure, one can distinguish common time slots for three colors when optimal efficiency of the SLM is achieved. This is crucial from the point of view of practical applications in color holography and image projection based on diffraction of light.

In the final part of the experiment we used a set of photodiodes and a Hameg HM507 oscilloscope to measure the absolute values of the diffractive efficiency in a direct and precise way. The used setup is presented in Fig. 4.

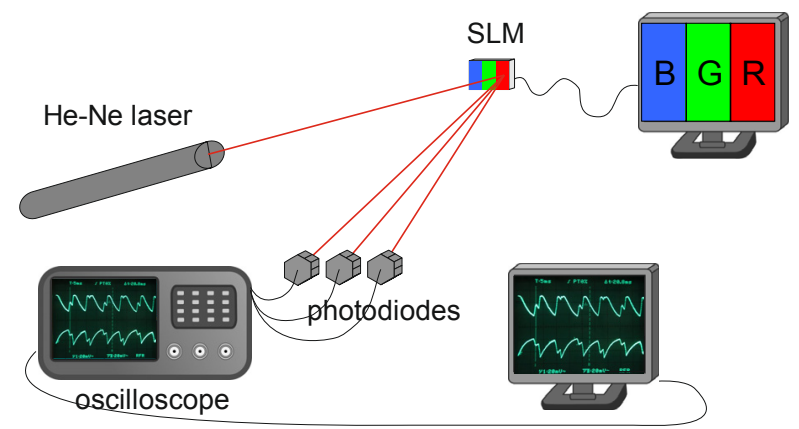

Fig. 4. Optical system for measuring the diffractive efficiency of diffractive gratings displayed on the SLM using a set of photodiodes and an oscilloscope.

Three laser beams of primary colors $(\mathrm{R}, \mathrm{G}, \mathrm{B})$ were reflected from appropriate parts of the SLM and focused on a set of photodiodes with a lens of $\mathrm{f}=50 \mathrm{~cm}$ (not shown in Fig. 4). By displaying periodical diffractive gratings on the SLM, additional light spots were formed from different diffractive orders. Exact locations of the light spots were established with the use of a matt plate. The active regions of photodiodes were placed exactly in the measured positions. The photodiodes were sensitive to each of the used wavelengths. They were connected to the oscilloscope at the same time in order to collect the time characteristics for a number of diffractive orders at the same time. Hence the calculated diffractive efficiencies had a lower systematic error. The data from the oscilloscope were collected through a GPIB interface and 
a program written in LabVIEW. The obtained results were in close agreement with the ones gathered using the CMOS camera. The detailed analysis of those results, including also the influence of the modulation depth on time characteristics of the SLM, will be further investigated.

As an example of the practical application of the presented method of SLM optimization, two photographs of a reconstruction from a Computer-Generated Fresnel Hologram (CGH) are given in Fig. 5 and Fig. 6. One can clearly see that the $0^{\text {th }}$ order and the inverted image associated with the $-1^{\text {st }}$ diffractive order are less visible in Fig. 6. This is due to the selective image acquisition moment, synchronized with the measured time characteristics of the SLM. One must take into account the fact that the full cancellation of the $0^{\text {th }}$ order is almost impossible because its energy is focused on a small area; therefore it forms a very bright spot. The holograms were reconstructed in a Fourier configuration, as depicted in Fig. 1.

To summarize, we have proposed a very simple technique of achieving optimal performance of an LCoS Spatial Light Modulator for three wavelengths corresponding to primary colors. The technique allows to synchronize the phase modulation fluctuations for three wavelengths collectively. This is achieved for the price of a slightly lower efficiency for each wavelength treated separately. We have shown the usefulness of this technique for improving the diffractive efficiency of diffractive gratings and Computer Generated Holograms. The method of optimizing of the SLM can be successfully implemented with standard laboratory equipment, i.e. a digital camera, or with greater accuracy using a set of photodiodes. The second option is more promising due to a lower error rate and more detailed information of the fluctuations of light intensity in different orders of diffraction. Moreover, the use of photodiodes increases the dynamic range of measured light intensity, compared to a $\mathrm{CCD} / \mathrm{CMOS}$ matrix.

The authors would like to thank HOLOEYE Photonics AG for a valuable support. This work has been supported by the European Union in the framework of European Social Fund through the Warsaw University of Technology Development Programme.

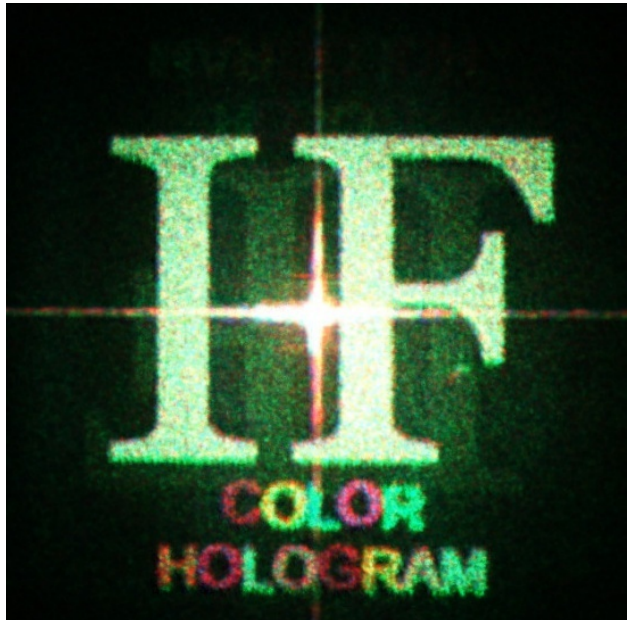

Fig. 5. Experimental reconstruction of a colour Fourier hologram with a $1 / 30$ s exposure. Disturbing $0^{\text {th }}$ order and inversed image from $\left(-1^{\text {st }}\right)$ order.

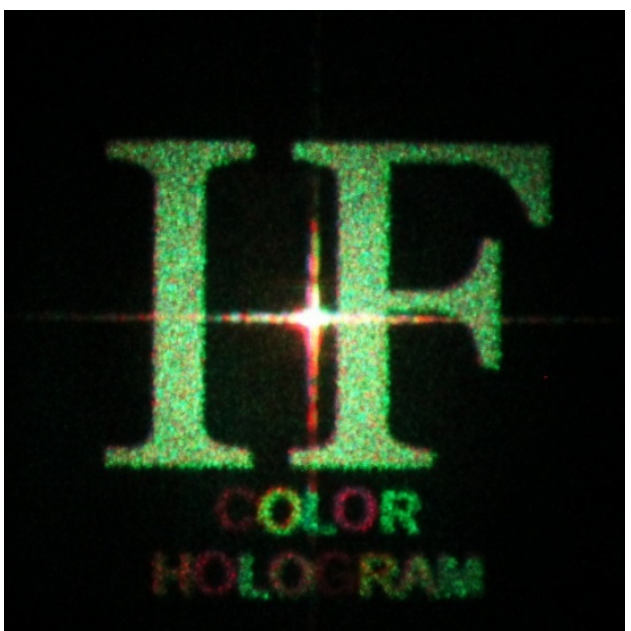

Fig. 6. Experimental reconstruction of a colour Fourier hologram with a 1/1000s exposure matched with SLM fluctuations characteristics. Suppressed $0^{\text {th }}$ order and inversed image from $\left(-1^{\text {st }}\right)$ order.

\section{References}

[1] M. Makowski, M. Sypek, A. Kolodziejczyk, Opt. Expr. 16, 11618 (2008).

[2] M. Makowski, M. Sypek, I. Ducin, A. Fajst, A. Siemion, J. Suszek, A. Kolodziejczyk, Opt. Expr. 17, 20840 (2009).

[3] M. Makowski, I. Ducin, M. Sypek, A. Siemion, A. Siemion, J. Suszek, A. Kolodziejczyk, Opt. Lett. 35, 1227 (2010).

[4] S. Osten, S. Krüger, A. Hermerschmidt, Proc. SPIE 6487, 64870X1 (2007).

[5] A. Lizana, A. Marquez, I. Moreno, C. Iemmi, J. Campos, M.J. Yzuel, JoEOS:RP 3, 08012 (2008). 